\title{
Comparative and functional study of integumentary system of two different reptiles: adaptation to their different modes of life
}

\author{
Samy A. Saber ${ }^{1}$, Boshra A. ElSalkh², Ali G. Gadel-Rab ${ }^{3}$, Fatma A. Mahmoud ${ }^{4}$, Asmaa A. El- \\ Dahshan $^{2}$, Doaa I. Gewily ${ }^{2}$ \\ 1Department of Zoology, Faculty of Science (Boys branch), Al-Azhar University, Cairo, Egypt \\ 2Department of Zoology, Faculty of Science (Girls branch), Al-Azhar University, Cairo, Egypt \\ 3Department of Zoology, Faculty of Science, Al-Azhar University, Assiut, Egypt \\ 4Department of Zoology, Faculty of Science, Assuit University, Assiut, Egypt
}

\begin{abstract}
Corresponding author: Doaa esmail,email: doaa_esmail2000@yahoo.com

Aim of the work: the present study aimed to illustrate the structure and function of the integumentary system of two reptile's species (Bosc's fringe toed-lizard (Acanthodactylus boskianus, A. boskianus) and spotted fan-fingered gecko (Ptyodactylus guttatus, P. guttatus) and discussed the influence of different behaviors on their structure (keratinzation and pigmentation, claws, adhesion pads and sensory hairlets). Material and methods: the present study used light and scanning electron microscopy to investigate the structure of skin layers of A. boskianus and P. guttatus. Results: the thin epidermal layer of Acanthodactylus boskianus was covered by hard keratinized horny epidermal scales, while the epidermal layer of Ptyodactylus guttatus was well distinguished and covered by soft keratinized layer. Moreover, in $P$. guttatus the scales were non-overlapping along the whole length of the body with dome-like shaped on head region, while in A. boskianus the scales were overlapping and articulating with each other by thin hinge. In the present study the dermis of A. boskianus, was the deeper layer of collagenous connective tissue with a rich supply of blood vessels and nerves, while, the dermis of $P$. guttatus was formed of both collagenous and adipose tissues. Three types of chromatophores (melanophores, iridophores and xanthophores) were distinguished in the skin of both studied species. In A. boskianus, the melanophores were black, large and have dendrites invaginates in the stratum basale of epidermis. The iridophores (reflecting pigments) were scattered in the dermis with large number in the ventral skin than the dorsal one. In P. guttatus, melanphores they were larger, blackish brown and scattered within dermis beneath the iridophores with less number in P. guttatus than that of A. boskianus. Xanthophores (absorbing pigment) were more prominent in P. guttatus than that of $A$. boskianus. Conclusion: examination of the skin of the two studied reptile species revealed great variations in distribution of chromatophores through the whole body reflecting the adaptation of each animal to different modes of life.
\end{abstract}

Keywords: skin, scales, chromatophores, foraging activities, reptiles

\section{Introduction}

Skin is phylogenetic source of surface specialization which reflects a greater commitment of animals to a terrestrial existence ${ }^{[1]}$. Perhaps the focus of evolutionary events in the integument has been as much on this interaction as on layers themselves. The epidermal-dermal interaction is evident even in tissue transplants between species from different classes. Reptiles represent the first amniotes. From stem reptiles, extant reptiles, birds and mammals have evolved. When reptiles established themselves on land, their integument can adapt to the challenges of terrestrial life, developing a multi-layered stratum corneum which is capable of barrier function and protects the body from abrasion, dehydration, microbial infections and ultraviolet protection. For better mechanical protection, diverse reptilian scale types have evolved ${ }^{[2]}$. Starting from these basic needs, different types of reptilian scales are evolved in the Mesozoic period to serve different functions [3] and adapt diverse species to the different niches. The pigmentation on the integument of animals forms one of the most dramatic and conspicuous biological patterns. The epidermis of lepidosaurians (lizards, snakes, and sphenodontids) is of particular complexity and interest ${ }^{[4]}$. It has very colourful scales and is organized in an attractive pattern with hard (beta) and soft (alpha) layers ${ }^{[2]}$. A scaly, keratinized integument is one of the characteristic features that airproof the skin of reptiles ${ }^{[4]}$. In the dermal skin of reptiles four 
basic types of pigment cells have been recognized: xanthophores, erythrophores, iridophores, and melanophores ${ }^{[5]}$. The colour intensity as well as the patterns of the skin in different reptilian species vary according to the distribution of epidermal melanocytes and dermal melanophores, lipophores (xantophores and erythrophores) and iridophores ${ }^{[6]}$. The spatial arrangement and architectural combination of these pigment cells can produce a multitude of skin colors in reptiles ${ }^{[5]}$.A study described the histology, structure, and the physiological importance of the squamate integument ${ }^{[2]}$.

Integument sense organs were firstly described for reptiles by Leydig ${ }^{[7]}$ in the lizard's genera Lacerta and Angus and in the snake genus Coronella. The author described small depressions in the surface of their scales and considered them as "organs of the $6^{\text {th }}$ sense" and compared them with the taste buds of fishes and amphibians. Scortecci ${ }^{[8]}$ examined in details the receptors of Agamids and Iguanids species. Crotaline and Boid snakes possessed infrared imaging receptors called pit organs, which work along with their visual and other sensory system to enable them to detect, locate, and apprehend prey ${ }^{[9]}$. Little is known about the presence and structural pattern of these thermoregulatory organs in lizards. The receptors possessing hair-like structure were described by many authors under different names (hair-like structures, setae-bearing organs, receptors with bristles and so on). Landmann ${ }^{[10]}$ described six different types of skin receptors; one with bristles and others without. De Haan ${ }^{[11]}$ reported the presence of small sense-organ like pits on the top of the head of several Psammophine snake species including: Dromophis lineatus, Malpolon monspessulanus, several Psammophis species and Rhamphiophis rubropunctatus. The present work aimed to investigate the structure of skin and characterization of sensory organs at the dorsal and ventral skin surface of two lizard species with different habits (Acanthodactylus buskianus and Ptyodactylus guttatus).

\section{Materials and Methods:} Experimental animals:

Fourteen adult Bosc's fringe-toed lizard, Acanthodactylus boskianus and spotted fan-toed geckos, Ptyodactylus guttatus were collected from different regions of Sinai and sacrificed by ether anesthesia. For light microscopic investigations, some specimens were fixed in $10 \%$ neutral formalin for two days and prepared for paraffin embedding; serial sections of $7 \mu \mathrm{m}$ were prepared and then stained with Haematoxylin and Eosin, Masson' trichromic stain and periodic acid Schiff reagent (PAS-reaction) ${ }^{[12]}$. U3cmos $14000 \mathrm{Kpa}$ (usb2.0) model of camera were used to take the photos. For scanning electron microscopy specimens were fixed in 5\% glutaraldehyde in a cacodylate buffer for $48 \mathrm{hr}$ at $4^{\circ} \mathrm{C}$ and washed in three changes of $0.1 \%$ cacodylate buffer, then the specimens were post- fixed in a cacodylate buffered solution of $1 \%$ osmium tetroxide for $2 \mathrm{hr}$ at $37^{\circ} \mathrm{C}$. The specimens were washed in the same buffer three times, dehydrated and then infiltrated with amyl acetate for two days. The drying of specimens is accomplished by the critical point drying using liquid $\mathrm{Co} 2$, mounted and sputter-coated with gold. The specimens were examined on a Jeol scanning electron microscope (J S M$5400 \mathrm{I} \mathrm{V}$ ), at $15 \mathrm{kv}$.

Aim of the work:

The present study deals with the examination of the integumentary system by light microscopy (LM) and scanning electron microscopy (SEM) of two adult lizards Acanthodactylus boskianus and Ptyodactylus guttatus collected from different regions of Sinai attempting to correlate the relationship between structure of the skin and their derivatives and the foraging habits of the two selected squamate species which differ in their classification as well as foraging strategy for feeding.

Results

In both studied reptile species, the skin was investigated along the whole body and on limbs, under the light and scanning electron microscopes. The epidermal and dermal layers exhibited skin structure in vertical sections.

\section{Histological investigation of body skin}

In A. boskianus, histological investigation of dorsal and ventral skin revealed that skin along the whole body was covered by flattened overlapping epidermal scales, while scales on the head region were dome-like shape tubercles being wider more than longer (Figs.1A, 2A, 2 B). Skin of both dorsal and ventral surfaces was composed of two principal layers; the epidermis and dermis which were followed by subcutaneous and muscle layers. In $A$. boskianus, the layers of epidermis were thin and 
difficult distinguished. Under high magnification we observed that the epidermis was composed of one or two nucleated layers; stratum basale and stratum granulosum. The stratum basale had cubodial cells with oval and rounded nuclei which lie on the basement membrane. The stratum granulosum constitutes the middle transitional layers which appear in some regions of the body, but not distinguished in the head region (Figs. 1A, 2B). The stratum granulosum was composed of 1-2 nucleated layers which were flattened superficially to forming the stratum corneum that was covered by hard keratin to form the horny epidermal scales (Figs. 1B, 2B). The horny scales were connected with each other by articulating membrane which was considered as a hinge between the epidermal scales. The thickness of epidermis of both dorsal and ventral surfaces was obviously thicker on the exposed scale surfaces than that in the hinge regions (Figs. 1A, 2B). The dermal layer was located beneath the epidermis that was formed of fibrous connective tissue fills with dense collagenous fibers and different types of chromatophores. Histological investigation of the skin of $A$. boskianus revealed that the outermost area of dermis of ventral skin consisted of abundant accumulation of iridophores (reflecting cells) more than that of the dorsal skin (Figs. 1A, B). The iridophores contained brownish-yellow granules and arranged in horizontal position adjoining to basement membrane of the epidermis. Moreover, it was observed that the appearance of melanophores underlie the iridophores which appeared as black cells are scattered in the dermis. Some melanophores had dendrites extended into the stratum basale of epidermis (Figs.1A, 2B).Meanwhile, the epidermis of skin of $P$. guttatus, was composed of multilayered cells; the basal layer (stratum basale) which formed the base of the epidermis with oval nucleated cells which generated to form one layer of polyhedral cells with rounded nuclei (stratum granulosum). The cells of stratum granulosum were flattened and converted into squamous type with oval-shaped nuclei that dies to form the keratin layer (Fig.1D).The thin keratinized layer covered both the dorsal and ventral epidermis forming small hock and dome-like shaped epidermal scales which were separated by thin inter-scale hinge (Fig. 1C). The dermal layer of both the dorsal and ventral skin of $P$. guttatus along the whole body length was divided into three layers: outer and inner adipose tissue sandwiching middle compact fibrous one. Moreover, the adipose layer in the dorsal surface of head region was represented only by the outer one while is disappeared in the ventral surface (Fig. 1C). The dermal layer of the dorsal skin contained many voluminous melanophores which possessed multidendrites. The melanophores appeared as a blackish-brown cells which were aligned along the adjoining basement membrane of the epidermis in some regions and were scattered in the dermis overlies the iriodophores and Xanthophores in other ones (Figs. 1C, 2C, 2D).

\section{SEM and Histological investigation of} limb's skin

Both the fore and hind limbs of the two investigated species; A. boskianus and $P$. guttatus had five fingers and toes. In $A$. boskianus their fingers and toes end by pointed claws which had central oval pit in their ventral surfaces that shown well in SEM investigation (Fig. 3D). Moreover, fore/hind limbs of $A$. boskianus were covered by modified overlapping scales which exhibited variation in size from periphery to the central region of the ventral surface of both manus and pes (large, median, and newer small scales) (Figs. 3A,B). Distally, on their fingers and toes these scales become wide and large (Fig. 3A). SEM investigation of limbs of $A$. boskianus revealed that these scales had leaf-shaped with pointed distal tip and provided with transverse ridges on its surface (Fig. 3C). Histological investigation of the scales of fore/hind limbs showed that the epidermal layers were the main constituent of these scales that similar to the structure of scales on the whole body length with presence of sensory hairlet bristles located in hinge area of scales (Fig. 3E) as well as the dermal layer was filled with dark brown melanophores and iriodophores (Figs. 3 E, F).

In $P$. guttatus both fore/hind limbs were covered by polygonal, bud shaped scales those were larger and wider on the ventral surface than dorsal one (Fig. 4C). SEM investigation of fore/hind limbs of $P$. guttatus exhibited scales pear groups of three hairs of sensory organs known as pits with sensory hairlet bristles located in hinge area of some scales and at the distal terminal apex of others. The epidermal sense organ appeared in the form of lenticular pit had a delicate funnel-like 
structures emerging above the scale surface. The peripheral edge of the sense organs was sharply delineated and the rim was concave on the inner surface forming longitudinal furrow (LF). Each sensory pit showed the attachment of one or more hairlet bristles (Fig. 4C). Histological investigation of limbs of $P$. guttatus exhibited that these hairlet bristles were extension of keratin layer lay on pit that formed by invagination of epidermal layers like that of A. boskianus (Fig. 3E).However, the ventral surface of the whole length of fingers and toes of $P$. guttatus were provided with adhesion pads which were arranged proximally in transverse direction and distally these pads rearranged in radial direction (Figs. 4 A, B, D). SEM investigation of adhesion pads of $P$. guttatus revealed that these pads were formed of clusters of setae (Fig. 4B). The histological investigation of adhesion pads exhibited the same structure of epidermal layers that covered the body skin. The stratum granulosum of epidermis of body skin were flattened to form the stratum corneum. While, stratum granulosum of epidermis of both manus and pes were elongated forming columnar cells with rounded nuclei filled with keratin granules in their apical portion (Figs. 4E, G). Meanwhile, the superficial layer of stratum granulosum of tip of fingers and toes was differentiated into multilayer of cells lack their nuclei and fill with keratin granules. Superficial to these cells, two nucleated layers were observed which protruded on the surface of adhesion pads constituting the setae (Fig. 4F).

\section{Figure legends}

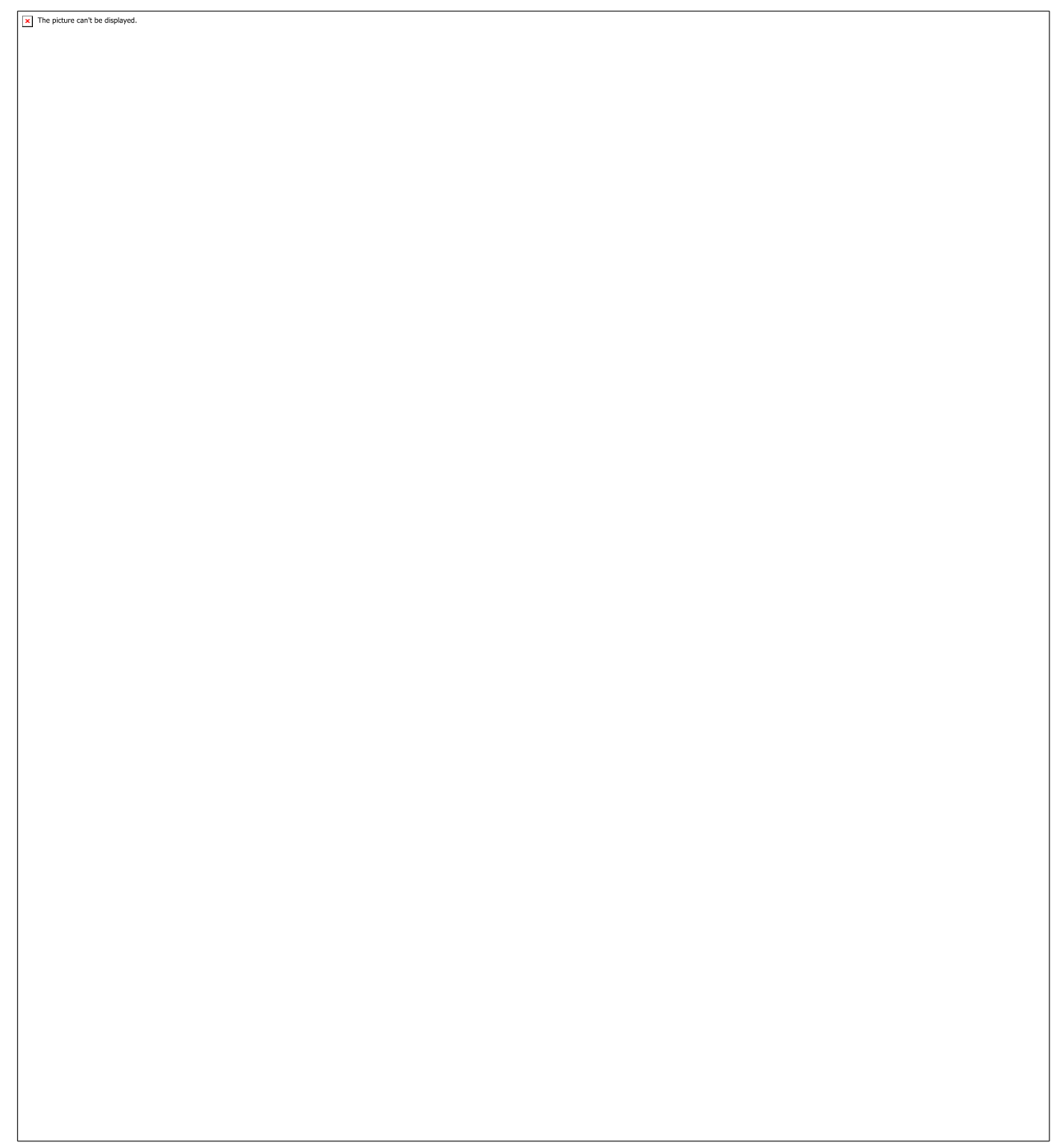


Fig.1. Transverse section through head (A, dorsal surface and B, ventral surface of the A. boskianus, $\mathrm{x} 400)$ and $(\mathrm{C}$, dorsal surface, and $\mathrm{D}$, ventral surface of the P. guttatus, Cx100, x400 and D, x400, $\mathrm{x} 1000$ ) showing DE, dorsal epithelium, VE, ventral epithelium, dom-like shape (double arrows), interscale hinge ( arrow), stratum basale (arrowhead), stratum granulosum (white arrow), stratum corneum (yellow arrow), collagen fibers in dermis( c), adipose connective tissue (star), melanophores (M), iridophores (I) and xanthophores (X). (Masson's trichrome stain).

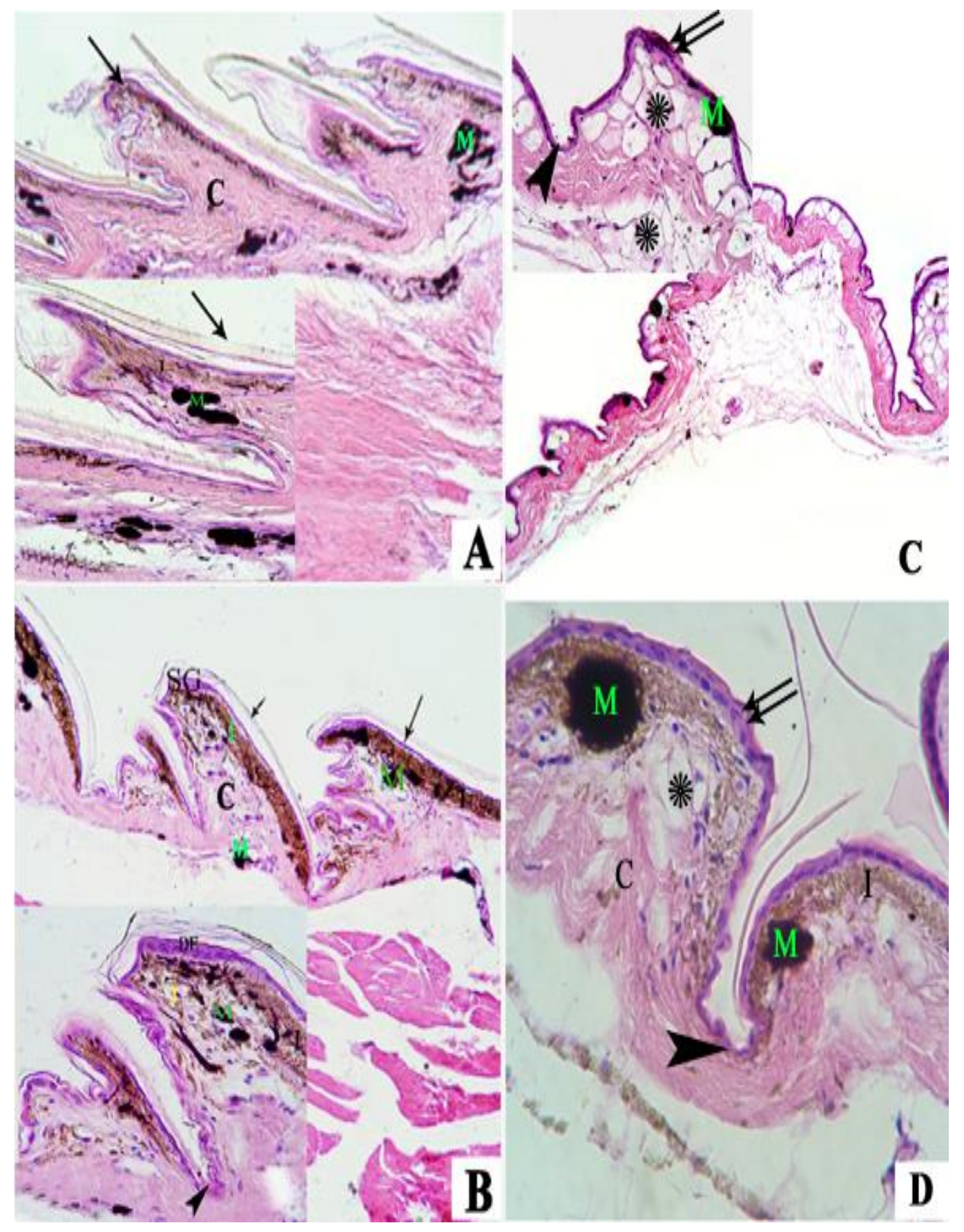

Fig.2. Transverse section through body (A, dorsal surface and B, ventral surface of the A. boskianus, $\mathrm{x} 100, \mathrm{x} 400)$ and $(\mathrm{C}$, dorsal surface, and $\mathrm{D}$, ventral surface of the P. guttatus, x100, x400) showing flattened overlapping scale (arrow), dom-like shape (double arrows), inter-scale hinge (arrowhead), collagen fibers in dermis (c), , melanophores (M), iridophores (I) and xanthophores (X). (Haematoxylin and Eosin stain). 


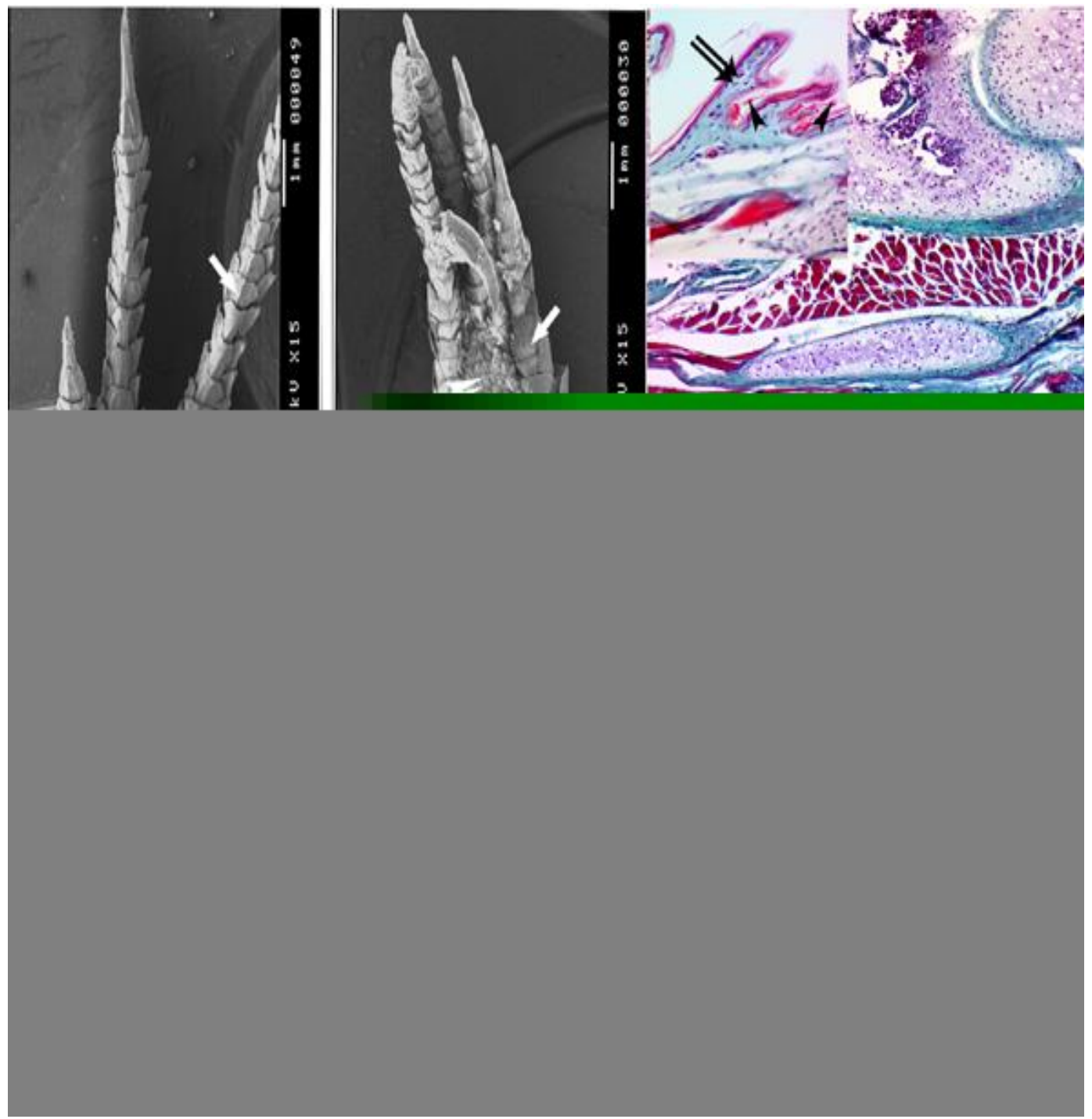

Fig.3. (A and B) Scanning electromicrograph of fore limb and hind limb of A. boskianus respectively showing large scale (white arrow) and small scales in central of ventral surface of pes (white double arrowhead), central oval pit on its claw (arrow) leaf-shaped scale (whit double arrows) and transverse ridges on the surface of scale (white arrowhead). (C) Longitudinal section through finger of $A$. boskianus showing flattened overlapping scale (double arrows) and inter-scale hinge (black arrow head), E. x100 and F. x400.(D) High magnification of the dorsal skin of fore limb A. boskianus showing dorsal epithelium (DE), oval nucleus of stratum basale (yellow arrowhead) melanophores (M), xanthophores (X) and collagen fibers in dermis ( C), x1000. (Masson's trichrome stain). 

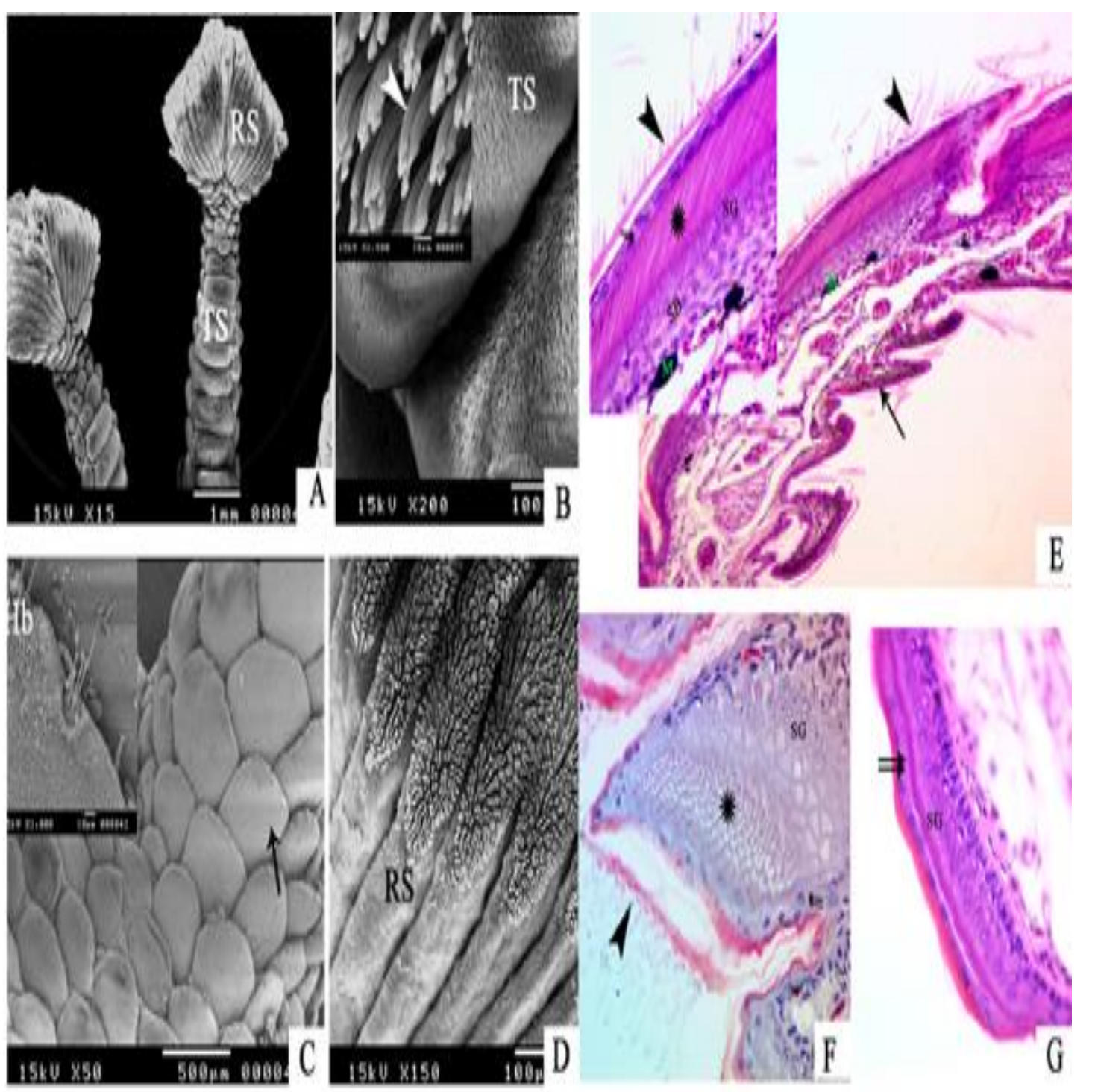

\section{Fig. 4}

Fig.4. (A and B) Scanning electromicrograph of finger of $P$. guttatus showing transverse adhesion pad (TS), radial adhesion pad (RS), overlapping scale (arrow), hairlet bristle on terminal apex of scale (Hb) and cluster of setea (arrow head) (E, F and G) Longitudinal section through adhesion pads on finger of A. boskianus showing stratum basale (SB), stratum granulosum (SG), cells fills with keratin granules (star), setae cells (double arrows), and setae (arrowhead), x400. (Masson's trichrome stain).

\section{Discussion}

Skin represents the first line of defense against disease. Although it preserves different functional activities in avian and mammalian organism, reptiles have extraordinary structures facilitated their living in hard terrestrial environment ${ }^{[13]}$. The skin of two studied species; A. boskianus and $P$. guttatus had some variations related to its protection against hard terrestrial life and mechanical shields which can prevent water loss during expose to ultraviolet (UV) irradiation. Both studied species depend on increasing of keratinization and pigmentation of dorsal and ventral skin. A. boskianus (Lizard) is diurnal insectivorous species forage in open area under direct sun-lights with sparse 
vegetation and very hot sands adopting active search strategy to find food. While, P. guttatus (Gecko) is a diurno-nocturnal omnivorous species, feed on insects adopting "sit and wait" strategy and has the ability of climbing on trees, rocks and vertical surfaces. Skin of the two species was composed of two typical layers, as in other reptiles, the outer epidermis and the inner dermis. The thin epidermal layers of $A$. boskianus were covered by hard keratinized horny epidermal scales, while the epidermal layers of $P$. guttatus were well distinguished and covered by soft keratinized layer. The composition of epidermal layers of $A$. boskianus adopted to protect the animal against water loss and offer protection from abrasion in the terrestrial area and as mentioned in the skin of lizards and snakes ${ }^{[14,15]}$. This result is in accordance with those of Alibardi ${ }^{[16]}$ who reported that the outer surface of lizards and snakes was consisted of a strongly cornfield epidermis, which provided stiffness for the scale. While, the composition of epidermal layers of $P$. guttatus were accommodates to high pressure during scape under rocks from predation. Moreover, in P. guttatus the scales were non-overlapping along the whole length of the body with dome-like shaped on head region, while in $A$. boskianus the scales were overlapping and articulated with each other by thin hing. This arrangement of epidermal scales on the body of the two studied species facilitates the mobility of body during food foraging ${ }^{[17,18]}$. Complex skin pigmentation patterns are exhibited by various vertebrate animals. The distribution of skin pigments is the main factor which determines the ultimate pigmentation pattern of a species ${ }^{[19]}$. In the present study the dermis of A. boskianus, was the deeper layer of collagenous connective tissue with a rich supply of blood vessels and nerves, while, the dermis of $P$. guttatus was formed of both collagenous and adipose tissue. The collagenous tissue acts as a barrier of water loss and solar radiation that was more distinguished in the dermis of $A$. boskianus. The adipose tissue which was represented by two layers in the skin of the nocturnal $P$. guttatus serves as heat reservoir ${ }^{[17}$, 18]. Three types of chromatophores; melanophores, iridophores and xanthophores were distinguished in the skin of both studied species. As it was the case with other species of reptiles and amphibians in the results of ${ }^{[20]}$ and in A. orientalis in the results of ${ }^{[21]}$ who added that the chromatophores were not organized in a functional chromatophoric unit.In $A$. boskianus, the melanophores were black, large and have dendrites invaginates in the stratum basale of epidermis. The iridophores (reflecting pigments) were scattered in the dermis with large number in ventral skin than the dorsal one. In $P$. guttatus, melanphores were larger, blackish brown and scattered within dermis beneath the iridophores which was with less number in $P$. guttatus than that of A. boskianus. xanthophores (absorbing pigment) were more prominent in $P$. guttatus than that of $A$. boskianus in agreement with the result of ${ }^{[21,22]}$ The variation in distribution of chromatophores within the dermal layer of skin of both studied species allow them to blend into the background of their natural eniverment (camouflage) as a defense mechanism from the major predators e.g. birds and other reptiles both of which have well developed color vision. Moreover, numerous Iridophores (light- reflecting cells) was observed in ventral skin of A. boskianus more than that in $P$. guttatus. This type of pigmentation may be reflecting the rays that radiate from ground during the foraging of $A$. boskianus in open area at day light. Meanwhile, $P$. guttatus (Gecko) is a diurno-nocturnal species mostly active at night and requires absorption of energy that reflected from the ground by the xanthophores (light-absorbing pigment cells) in its ventral skin than that in $A$. boskianus. However, the previously mentioned results confirmed that the scale morphogenesis and different types of chromatophores of the two studied species reflect a great adaptation to environmental habitat and foraging habits. Meanwhile, in the present study we observed another adaptation in these studied species, each of species possesses different tools for walking to facilitate searching about their prey. In $A$. boskianus, their limbs end by curved and pointed claws that aids in burrowing in sand and allow walk freely and rapidly to catching their prey or escape from predators. It's holding ability increase by presence of pits in ventral surface of claw. While, the P. guttatus, their limbs possessed adhesion pads at the distal end of finger and toes. These pads exhibited different direction; transversally on finger or toes and radially on its distal end. Moreover, that pad was formed of cluster of seta. The different arrangement of cluster of setae on the finger of $P$. guttatus gave easily attach and 
detach from the surface thereby it acted as adhesion pads allow climbing on vertical surfaces. Our study also demonstrated that there were two nucleated cells at the base of setae which acted as setae cells. These setae cells were differentiated from the superficial layer of stratum granulosum of fingers and toes of $P$. guttatus. The deformations of setae cells produce bioelectrical changes that important in sensation of the external stimuli and play a role in attachment and detachment of gecko. Guo et $a{ }{ }^{[23]}$ discussed the theory of the mechanism of adhesion of millions of setae on the toes of geckos and provided new ideas for designing and fabricating synthetic setae. Moreover, in the present study we detected epidermal sensory organs (hairlet bristles) on the terminal apex of scales that covering the fingers and toes of $P$. guttatus. These hairlet bristles acted as mechanoreceptors may increase sensitivity of gecko to any external stimuli beside the setae. Ananieva et al. ${ }^{[24]}$ mentioned that it serves several functions as mechano and thermoreceptors and possibly sensitivity to humidity. El-Sayyad et al. ${ }^{[25]}$ recorded that the emergent of sensory hairlet structure with the advancement of growth facilitated in performing mechanical and sensory functions to accommodate with their habitat. Darwish [17] reported that the dorsal and ventral small scale of $P$. guttatus possessed two epidermal lenticular sense organs located its distal peripheral apex.

In conclusion, examination of the skin of the two studied reptile species revealed great variations in the pattern of distribution and arrangement of scales along the whole body surfaces. Also, claws, adhesion pads and sense organs along the fore/hind limbs and their fingers and toes exhibit great variation and act as tools for walking on land or adhesion on vertical surfaces of each species reflecting the adaptation of each animal with its foraging activities.

\section{References:}

1. Kardong K V (2009): Vertebrates: Comparative Anatomy, Function, Evolution. $7^{\text {th }}$ ed., Boston: McGrawHill, Amazon. P.816.

2. Chang $\mathbf{C}, \mathbf{W u}$ P, Baker $\mathbf{R}$ E, Maini $\mathbf{P}$ $K$, Alibardi $L$ and Chuong $C$ (2009): Reptile scale paradigm: Evo-Devo, pattern formation and regeneration. Int. J. Dev. Biol., 53: 813-826.

3. Chuong $\mathrm{C} M$, Nickloff B J, Elias P M, Goldsmith L A, Macher E, Maderson $\quad P$ A and Sundberg $J$ $\mathbf{P}$ (2002): What is the 'true' function of skin. Exp. Dermatol., 11: 159187.

4. Hildebrand M and Goslow G (2001): Analysis of Vertebrate Structure $\left(5^{\text {th }}\right.$ ed). John Wiley and Sons, New York.

5. Kuriyama T, Mryaj I K, Sugimoto $M$ and Hasegawa $M$ (2006): Ultrastruc ture of the dermal chromatophores in a lizard (Scincidae: Plestiodon latiscutatus) with conspicuous body and tail coloration. Zool. Sci., 23: 793-799.

6. Alibardi L (2013): Observations on the ultra-structure and distribution of chromatophores in the skin of chelonians. Acta Zool., 94: 222-232.

7. Leydig F (1868): Uber organe eines sechten sinnes nove. Acta Acad. Carol. Dresden, 34:1-108.

8. Scortecci G (1941): Recettori degli agamidi. Mem. Ital. Sci. Nat., 10: 209326.

9. Barrett $\mathbf{R}$, Maderson $\mathbf{P} \mathbf{F} \mathbf{A}$ and Maszler R M (1970): The pit organs of snakes. In: Biology of Reptilia Gans. C.(ed.). Academic Press, London, Pp:277-314.

10. Landmann L (1975): The sense organs in the skin of the head of squamata (reptilian). Israel $\mathrm{J}$. Zool., 24: 99-135.

11. De Haan CC (2003): Sense-organ-like parietal pits found in Psammophiini (Serpentes, Colubridae). Comptes Rendus Biologies, 326: 287293.

12. Drury $\mathbf{R} \mathbf{A}$, Wallington $\mathbf{E} \mathbf{A}$ and Cancerson $R$ (1980): Carlton's Histopathological Techniques. $4^{\text {th }}$ Ed., Oxford University Press, Oxford, London, New York.

13. Russell P J W, olfe S L, Hertz P E, Starr $C$ and McMillan B (2008): Biology: the dynamic of science. Belmont, Calif: Brooks/Cole, England. p.145. 
14. Alibardi, L. (2003): Adaptation to the land: the skin of reptiles amniotes. J. Exp. Zool. Part B-Molecular and Developmental Evol., 298: 12-41.

15. Oufiero C E, Gartner G E A, Adolph $S C$ and Garland T J (2011): Latitudinal and climatic variation in body size and dorsal scale counts in Sceloporus lizards: a phylogenetic perspective. Evol., 65: 3590-3607.

16. Alibardi L (1998): Differentiation of the epidermis during scale formation in 192: 173-186. embryos of lizard. J. Anat.,

17. Darwish S T (2012): comparative light and ultrastrucural studies of skin in Stenodactylus petrii and Ptyodactylus guttatus (Reptilia: Gekkonidae). Egypt. J. Exp. Biol., 8(1): 9-14.

18. Szydlowski $\mathbf{P}$, Madej $\mathbf{J} \quad \mathbf{P}$ and Mazurkiewicz-Kania M (2017): Histology and ultrastructure of the integumental chromatophores in Tokay gecko (Gekko gecko) (Linnaeus, 1758). skin Zoomorphol., 136 (2): 233-240.

19. Bagnara J T and Hadley M C (1973): Chromatophores and color change. In: The Comparative Physiology of Animal Pigmentation. New Jersey, Prentice-Hall,Englewood Cliffs.

20. Bagnara $\mathbf{J}$ and Matsumoto $\mathbf{J}$ (2006): Comparative anatomy and physiology of pigment cells in non-mammalian tissues. In: Nordlund, J. J.; Boissy, R. E.; Hearing, V. J.; King, R.; Oetting, W. and Ortonne, P. J. (Eds.): The Pigmentary System, 2nd ed., Blackwell, Malden, MA. pp. 11-59.

21. Paray B A and Al-Sadoon M K (2017): Ultrastructure of the dermal chromatophores in the Fringe-toed lizard, Acanthodactylus orientalis. Zoologia,34:11923-11933.

22. Rossi J V (1996): Dermatology. In: Reptile Medicine and Surgery. Douglas R. Mader, editor, WB Saunders, NY., pp. 104-117.

23. Guo P, Liu Q, Xu Y, Jiang K, Hou M, Ding L, Yron RA and Burbrink FT (2012): Out of Asia: Natricine snakes support the Cenozoic Beringian Dispersal Hypothesis. Molecular Phylogenetics and Evol., 63: 825- 833.

24. Ananieva N B; Dilmuchamedov M E and Matveyeva TN (1991): The skin sense organs of some Iguanian lizards. J. Herpetol., 25(2): 186-199.

25. El-Sayyad H I, Yonis M W F, Bayomi F F M and Shalaby S (2009): Epidermal sense organs of the Gekkonid Tropicolotes tripolitanus Peters 1880. J. Cell Anim. Biol., 3(6): 088-092. 